\title{
Accuracy and adaptation of reaching and pointing in pitched visual environments
}

\author{
ROBERT B. WELCH \\ NASA-Ames Research Center, Mountain View, Califormia \\ and \\ ROBERT B. POST \\ University of California, Davis, California
}

\begin{abstract}
Visually perceived eye level (VPEL) and the ability of subjects to reach with an unseen limb to targets placed at VPEL were measured in a statically pitched visual surround (pitchroom). VPEL was shifted upward and downward by upward and downward room pitch, respectively. Accuracy in reaching to VPEL represented a compromise between VPEL and actual eye level. This indicates that VPEL shifts reflect in part a change in perceived location of objects. When subjects were provided with terminal visual feedback about their reaching, accuracy improved rapidly. Subsequent reaching, with the room vertical, revealed a negative aftereffect (i.e., reaching errors that were opposite those made initially in the pitched room). In a second study, pointing accuracy was assessed for targets located both at VPEL and at other positions. Errors were similar for targets whether located at VPEL or elsewhere. Additionally, pointing responses were restricted to a narrower range than that of the actual target locations. The small size of reaching and pointing errors in both studies suggests that factors other than a change in perceived location are also involved in VPEL shifts.
\end{abstract}

Under normal circumstances, perceived orientation of the body with respect to gravity is based on concordant information from the visual, vestibular, and proprioceptive systems (see Dichgans \& Brandt, 1978, and Howard, 1982, for reviews). The contribution of vision to this perception can be assessed by changing the orientation of the visual context with respect to any of the three body axes. For example, rotation or translation of the visual field in the yaw (i.e., left-right) dimension causes judgments of apparent straight-ahead to be shifted toward the side with the larger visual stimulus (Dietzel, 1924; Roelofs, 1935). Likewise, viewing contours that have been tilted counterclockwise or clockwise from vertical in the frontoparallel plane (i.e., roll) causes other stimuli to appear rotated in the direction opposite the tilt (Ebenholtz, 1977, 1985; Witkin \& Asch, 1948). Finally, visual displays that have been pitched downward or upward (top-forward or top-backward) cause, respectively, downward and upward shifts of the subject's visually perceived eye level (VPEL; Matin \& Fox, 1989; Stoper \& Cohen, 1989). In the study by Stoper and Cohen, for example, VPEL shifts of approximately $10^{\circ}$ occurred when subjects viewed the interior of a room that had been pitched by $\pm 20^{\circ}$.

We are grateful to Malcolm M. Cohen, Arnold Stoper, and Alan Hein for discussions in the development of these ideas. The assistance of Abigail Bautista, Ty Hoang, and Wendy Brunt is also gratefully acknowledged. Correspondence concerning this article should be addressed to NASA-Ames Research Center, Mail Stop 239-11, Moffett Field, CA 94035 (e-mail: robert_welch@quigate.arc.nasa.gov).
A variety of causes for the effect of visual pitch on VPEL have been proposed, including characteristics of the retinal image projection of pitched stimuli, extraretinal eye position information about the relation of eye to head, and perception of the direction of gravity (Matin \& Li, 1992; Stoper \& Cohen, 1989).

The viability (and relative importance) of these potential mechanisms can be investigated by measuring observers' accuracy in directing open-loop arm movements at targets placed within the pitched environment. If, for example, the upward VPEL shift obtained in an upwardly pitched room is based entirely on a downward shift in egocentric visual localization (whether from retinal or extraretinal information), commensurate errors of reaching too low for targets should occur. That is, subjects attempting to reach for a target placed at their VPEL should reach to approximately their true eye level, because that is where they have indicated that the target appears to be. If instead the pitchroom alters the observer's perception of the gravity vector (or the orientation of the whole body relative to gravity), minimal reaching errors might be expected, since such a change would apply equally to the seen location of the visual stimulus and to the felt position of the limb. That is, if the whole body is perceived to be rotated relative to gravity, a target may be perceived as being higher or lower as a result of pitch, but the subject would also reach correspondingly higher or lower.

The goal of Experiment 1 was to assess the accuracy of reaching with an unseen arm for targets presented at the subject's VPEL in a pitched visual environment. Ballinger 
(1988; Cohen \& Ballinger, 1989) measured target-pointing accuracy for subjects who were viewing the interior of a small pitched box and found errors that averaged about $50 \%$ of the VPEL shift. ${ }^{1}$ In the present study, we extended this technique to the much larger visual framework of a pitchroom. An additional issue that was addressed was whether subjects would undergo visual and/or visuomotor adaptation to the pitchroom when provided with visual feedback about their target-reaching accuracy.

\section{EXPERIMENT 1}

\section{Method}

Fifteen males and 9 females, $18-29$ years old, whose vision was either normal or corrected-to-normal (by contact lenses) served as subjects. All were right-handed paid volunteers from the local community and were experimentally naive. The pitchroom was $121 \mathrm{~cm}$ wide $\times 167 \mathrm{~cm}$ high $\times 279 \mathrm{~cm}$ long, and its base, when horizontal, was $58 \mathrm{~cm}$ above the floor. The pitch axis was located in the center of the room. The back and side walls, as well as the ceiling, were covered with a grid of $5.2 \times 5.2 \mathrm{~cm}$ squares. All observations were made with subjects standing inside the pitchroom at arm's length from the back wall.

VPEL measures. The initial VPEL measures were obtained with the pitchroom placed vertically. Subjects stood without head restraint and faced the inside back wall of the pitchroom as the experimenter projected a point of light from a low-power laser at a location randomly selected to be above or below subjects' true eye level as measured from the floor. The experimenter moved the point of light according to subjects' directions until it was reported to be at a given subject's VPEL. These measures were then obtained with the room pitched either $20^{\circ}$ up or $20^{\circ}$ down and finally with the room once again.vertical. All viewing was binocular, with subjects' vision of their bodies and the floor precluded by their proximity to the wall of the apparatus and instructions to look forward. Twelve subjects participated in the pitch-up condition and 12 in the pitch-down condition.

Reaching measures. After the initial determination of VPEL with the room vertical, subjects attempted to reach with the right index finger to the same level as a marker that had been placed on the wall at their VPEL. For these responses, the subject's view of the arm and hand was blocked by a curtain. The hand's starting position for each response was at the subject's side. The reaching response therefore involved both upward and forward motion of the arm. Subjects were directed to reach quickly, to maximize the ballistic component of the motion. This open-loop response was repeated 10 times, and its accuracy was recorded. Another set of 10 such responses were made and recorded after the VPEL had been measured with the room pitched ( $20^{\circ}$ up or $20^{\circ}$ down). Following these responses, subjects reached 20 times at the VPEL target in the pitched room with the curtain now retracted so that the tip of the finger could be seen as it touched the wall at the end of each response. This form of visual feedback, often referred to as "terminal exposure," has proved especially effective in producing adaptation to prismatic displacement (e.g., Welch, 1978). After the final VPEL measurement with the room vertical, subjects made 10 more open-loop reaching responses at the VPEL target.

\section{Results}

All VPEL and reaching measures were converted on the basis of individual subjects' heights and viewing distances to degrees of visual angle from objective eye level. In Figure 1, the mean of all subjects' VPEL measures during each phase of the study are shown for both pitch directions. Although VPEL was similar in magnitude for (a)

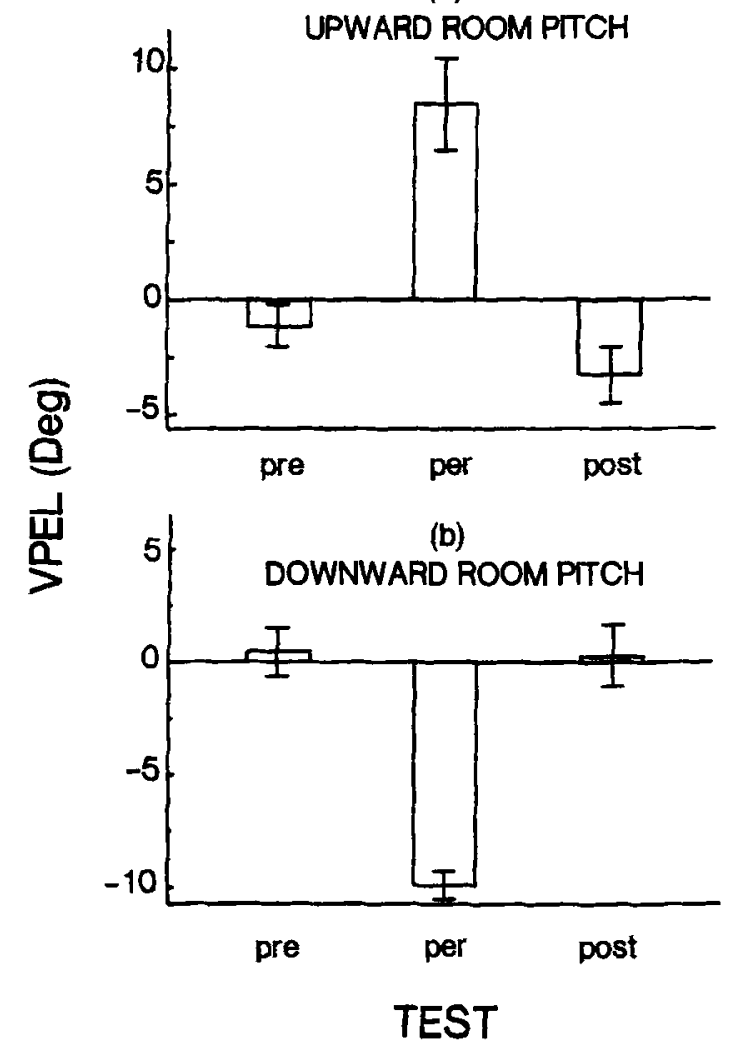

Figure 1. Mean VPEL measures prior to room pitch (pre), during room pitch (per), and following room pitch (post) in Experiment 1 for upward (a) and downward (b) room pitches. Error bars correspond to \pm 1 SD.

the two pitch directions in the pre- and postmeasures, it was shifted considerably upward and downward by upward and downward room pitch, respectively. The VPEL measures were analyzed by a 2 (pitch up/ pitch down) $\times$ 3 (pre-/per-/postpitch) analysis of variance (ANOVA). The main effect for pitch was statistically significant $[F(1,22)$ $=6.98, p<.05]$, as was the interaction $[F(2,44)=76.49$, $p<.001]$.

In Figure 2 are also found the VPEL measures, along with the mean response for each target-reaching trial for each of the three pitch phases of the study. It can be seen that in sessions with the upward room pitch (Figure 2a), subjects' open-loop reaching responses were close to VPEL during the prepitch measures. Immediately following room pitch, and with visual feedback still denied, subjects tended to reach below the measured VPEL. This relatively small error is consistent with the hypothesis that the VPEL shift induced by room pitch is based, at least in part, on change in the perceived location of visual stimuli. When visual feedback was introduced on the 21 st trial of the perexposure phase, reaching responses quickly became accurate with respect to the VPEL target. Finally, when the room was once again vertical, and in the absence of visual feedback, subjects reached above their VPEL. The pattern for the down- 
(a)

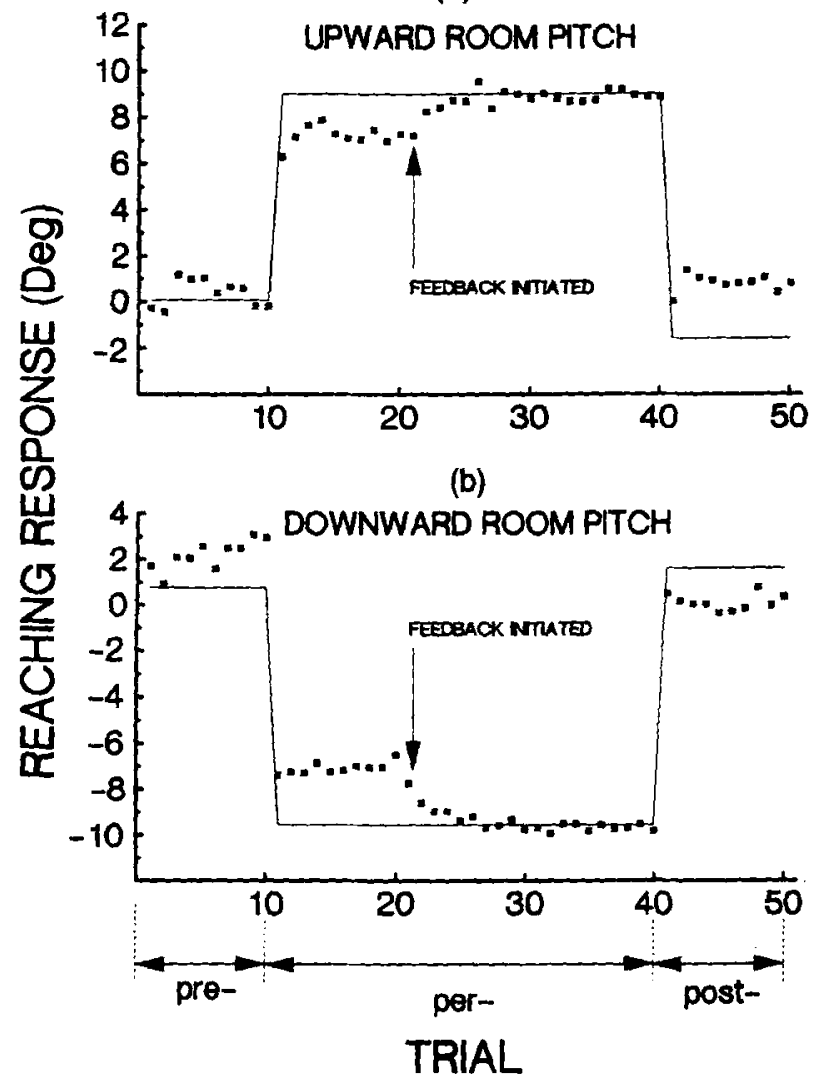

Figure 2. Mean location of reaching responses for each of $50 \mathrm{ex}-$ perimental trials (squares) for upward (a) and downward (b) room pitches in Experiment 1. The location of the VPEL to which subjects were attempting to reach is depicted by the solid lines. Arrows indicate the introduction of feedback on the 21 st reaching response.

ward pitch condition (Figure 2b) was similar to that of the upward pitch condition, but of course was reversed in direction in the per- and postpitch phases.

Reaching accuracy was statistically analyzed by first taking the difference between the reaching response and the location of the VPEL target. The mean of the 10 errors made by each subject during each of the three phases was determined separately for the two pitch-direction conditions. These means were analyzed by a 2 (pitch up/ pitch down $) \times 3$ (pre-/per-/postpitch phase) ANOVA. Neither pitch direction $[F(1,22)=.07, p>.1]$ nor phase $[F(2,44)=.56, p>.1]$ was statistically significant. The pitch direction $X$ phase interaction, however, was highly significant $[F(2,44)=28.61, p<.001]$.

In Figure 3 the data depicted in Figure 2 have been collapsed into the mean response for each of the three phases for each pitch direction. Once again, it can be seen that subjects reached lower than their upwardly shifted VPEL when the room was pitched up and higher than their downwardly shifted VPEL when the room was pitched down. The range of reaching responses of $13.9^{\circ}$ represented $75 \%$ of the $18.4^{\circ}$ range of mean VPEL values between the two pitch phases. Post hoc analyses confirmed that reaching errors were not significantly different for the pitch-up and pitch-down conditions during the prepitch measures $[F(1,22)=.6, p>.1]$, but were sig. nificantly different for the perpitch measures $[F(1,22)=$ $9.62, p<.01]$. The fact that the postpitch reaching errors were in the direction opposite the errors made during pitch is evidence of a negative aftereffect, the hallmark of adaptation to optically displaced vision (e.g., Welch, 1978). Post hoc comparisons confirmed that pre- and postpitch errors were significantly different from each other for both upward $[F(1,22)=9.93, p<.01]$ and downward $[F(1,22)=12.94, p<.01]$ pitch groups.

\section{Discussion}

Consistent with the results of previous research (e.g., Stoper \& Cohen, 1989), VPEL was strongly shifted in the direction of the room pitch (Figure 1). Furthermore, responses differed from true eye level when subjects were attempting to reach to their VPEL. That is, average VPEL was shifted from true eye level by about $9^{\circ}(45 \%$ of the room pitch), while subjects' open-loop reaching responses were about $2^{\circ}$ less than this (Figure 2). However, the latter errors were rapidly corrected (within five trials) when subjects were given terminal errorcorrective feedback about the accuracy of their responses (Perexposure Trials 21-30, as seen in Figure 2).

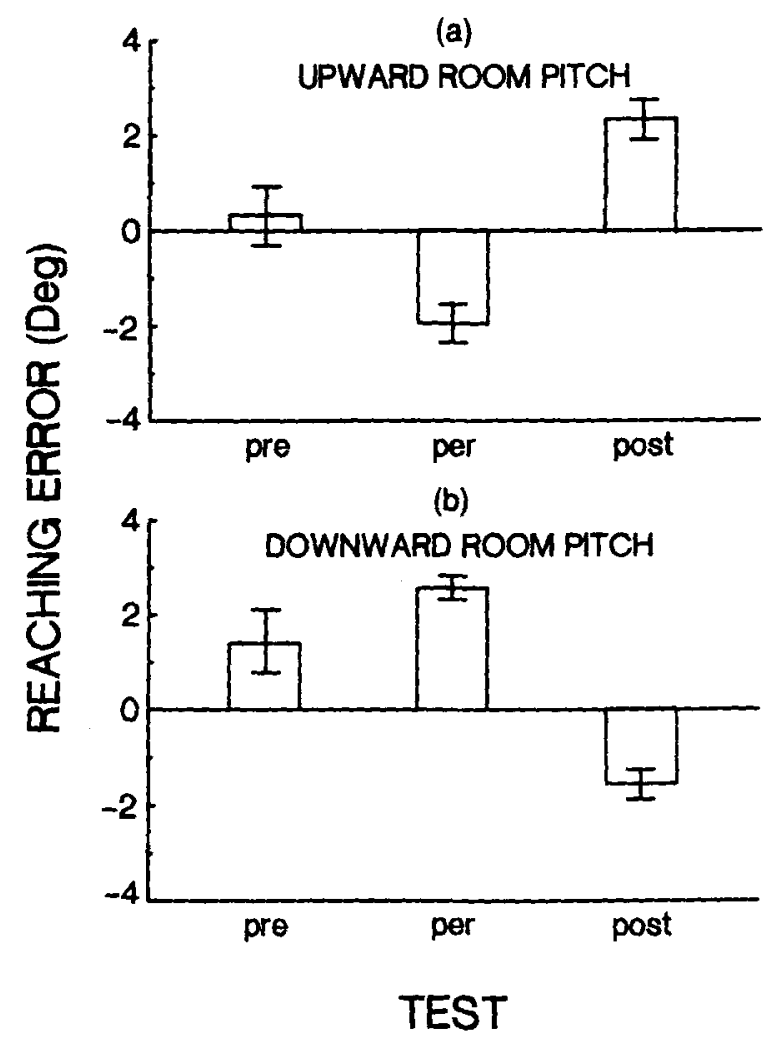

Figure 3. Mean reaching errors prior to room pitch (pre), during room pitch (per), and following room pitch (post) in Experiment 1 for upward (a) and downward (b) room pitches in Experiment 1. Error bars correspond to $\pm I S D$. 
When the pitchroom was once again set to vertical, subjects revealed negative aftereffects by erring in the direction opposite the errors they made when the room was initially pitched (Figure 3 ). It is interesting that the changes in reaching behavior occurred in the absence of any concomitant change in VPEL. For this reason, the changes of reaching during and after pitch are not the result of a change in the magnitude of illusory visual localization. Rather, the aftereffects are consistent with the hypothesis that subjects had recalibrated the linkage between their perceptual and motor spatial maps.

That the pitchroom caused target-reaching errors at all supports an interpretation that the VPEL shift results in part from a change in visual localization. However, if the VPEL shift were entirely explainable on this basis, subjects' reaching errors would have approximated it in size. The fact that they clearly did not indicates that this interpretation is incomplete. The finding that open-loop reaching errors are small in magnitude is consistent with the hypothesis that the VPEL shift is largely based on an alteration in the perception of the gravity vector. Such a change would apply equally to the seen location of the visual stimulus and the felt position of the limb and would therefore not be responsible for any reaching error. The finding of small reaching errors is therefore consistent with the possibility that both a small change in perceived visual localization and (by default) a larger shift in the perception of the gravity vector are contributing to VPEL shifts. If both factors were operating, reaching errors would represent a compromise between the patterns expected from the operation of either mechanism in isolation.

\section{EXPERIMENT 2}

The most important question raised by Experiment 1 is why subjects' open-loop reaching errors were so much smaller than their VPEL shifts. One possibility is that perception of the vector of gravity is altered by visual pitch, as previously discussed. Alternatively, it is possible that the VPEL shift reflects a combination of a small perceptual shift in the direction of the room pitch and a much larger nonperceptual (i.e., judgmental) shift in the same direction. The latter is analogous to the "straightahead shift" described by Harris (1974) for rotations of the visual field in the yaw dimension.

In the only deliberate test of the straight-ahead shift hypothesis of which we are aware, Harris and Gilchrist (1976) measured the effects of a yawed visual surround (the interior of a small box) on subjects' accuracy in setting a visual target straight ahead and pointing (openloop) both straight ahead and at visual targets located elsewhere in the box. Whereas the yawed surround biased both the setting of a visual target to straight ahead and pointing to straight ahead, it had no effect on the accuracy of pointing at the visual targets. On the basis of this pattern of responses, the authors concluded that subjects had undergone a change in their definition of straight ahead, rather than in visual localization or felt limb position. Had the latter changes occurred, openloop target-pointing responses would presumably have been affected as much as the straight-ahead measures.

The task which was investigated in our first experiment was formally identical to that studied by Harris and Gilchrist (1976), except that the axis of rotation of the surround about the observer was pitch rather than yaw. In Experiment 1, subjects were asked to reach for a visual target with an unseen limb, a condition similar to that of Harris and Gilchrist, who found no effect of the yawed surround on this measure. However, in our experiment the target to which subjects attempted to reach was coincident with the VPEL (analogous to the straight-ahead position), the condition for which Harris and Gilchrist had found an effect. It is therefore possible that the subjects in our experiment were trying to reach for VPEL because the target was positioned where they had just indicated VPEL was. Perhaps, then, this coincidence explains why reaching accuracy in our first experiment represented a compromise between the VPEL and true eye level.

Experiment 2 was undertaken to examine the possible contribution of the straight-ahead shift to the discrepancy between the VPEL and reaching measures that we obtained in Experiment 1. In addition to having subjects attempt to point at a target placed at the VPEL location, accuracy was measured for a target above and a target below VPEL. Because the latter two targets are not located at VPEL, they should be immune to judgmental shifts because such shifts are assumed to be specific to VPEL (as they are to straight ahead). As before, pointing was done with the unseen limb.

The presence of additional targets in Experiment 2 also permitted us to assess the accuracy of open-loop pointing under conditions in which no misperception of visual location is expected (i.e., when the room is upright). It is logically possible that the reason why subjects' reaching responses underestimated the measured VPEL shifts in Experiment 1 is a general tendency for subjects to "undershoot" during open-loop pointing (see, e.g., Nemire \& Bridgeman, 1987).

\section{Method}

Sixteen right-handed volunteers with normal or corrected-tonormal vision (contact lenses) were obtained from introductory psychology courses at the University of California at Davis to serve as subjects. Each subject participated in one experimental session, during which VPEL measures were obtained for each of three room orientations (vertical, pitch-up $20^{\circ}$, and pitch-down $20^{\circ}$ ). Following the determination of VPEL for a given room pitch, subjects made a series of pointing responses at targets placed at, above, and below their VPEL. Half of the subjects were tested in the following order: room pitch up, room vertical, room pitch down. The remainder were tested in the reverse order.

VPEL measures. VPEL measures were taken in a manner similar to that in Experiment 1. The pitchroom used in this experiment was $152 \mathrm{~cm}$ wide $\times 152 \mathrm{~cm}$ high $\times 182 \mathrm{~cm}$ long, and its base was suspended $50 \mathrm{~cm}$ above the floor when horizontal. The back and side walls were covered with alternating black and white vertical stripes, measuring 13.5 and $2.5 \mathrm{~cm}$ wide, respectively. Subjects sat in the center of the pitchroom with head position stabilized by a chinrest. 
Pointing measures. After the determination of VPEL for a given room orientation, subjects attempted to direct a small laser pointer at three markers on the back wall of the pitchroom. They held the laser in the right hand so that it protruded a short distance beyond the index finger. The markers were located at VPEL and $20^{\circ}$ above and $20^{\circ}$ below VPEL. For these responses, the subject's view of the arm, hand, and laser was blocked by a curtain. The pointing responses were similar to those made in Experiment 1, as subjects began each response with the arm at their side and were instructed to move it very quickly. Unlike in Experiment 1, however, subjects were out of reach of the wall of the apparatus. Five pointing attempts were made and recorded for each of the three markers for each of the three room orientations. The order of targets to which subjects pointed was pseudorandomly varied, with the constraint that no two consecutive responses were to be made for the same target.

\section{Results}

Average VPEL for each room pitch is presented as a symbol in Figure 4, together with symbols depicting the upper and lower target locations $\left( \pm 20^{\circ}\right.$ from VPEL). It is apparent in the figure that, as in Experiment 1, VPEL

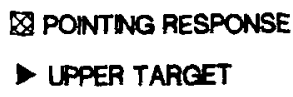

- VPE \& VPE TARCET
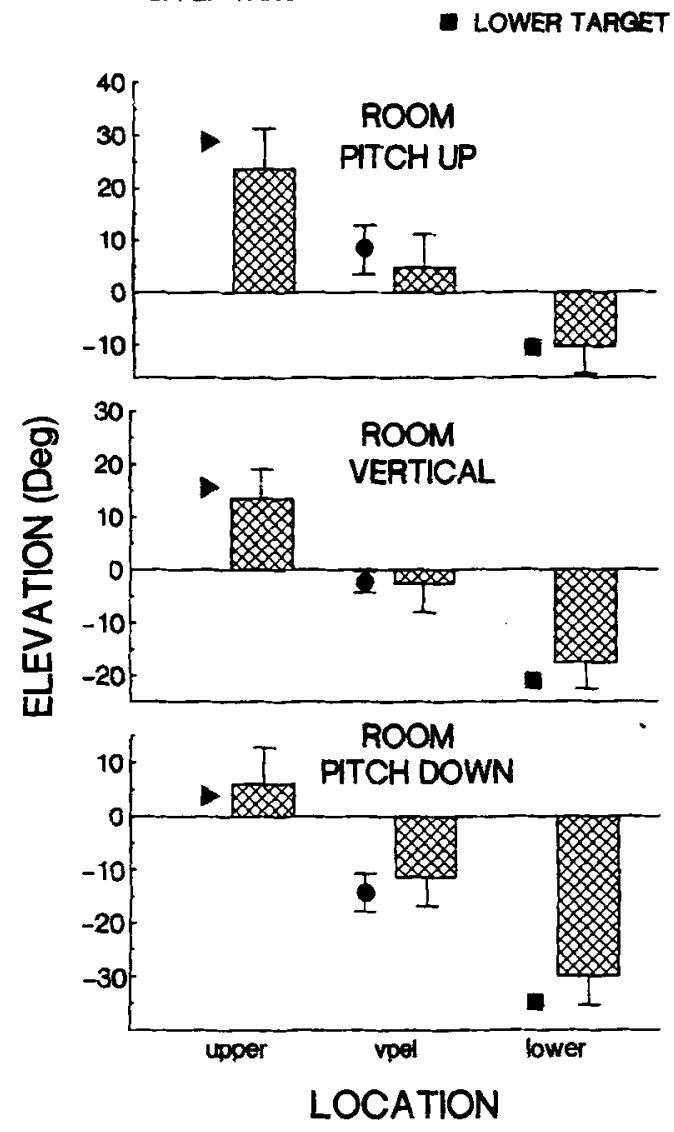

Figure 4. Circles depict mean VPEL response and target location in Experiment 2. Upper and lower target locations are represented by triangles and squares, respectively. Bars adjacent to each symbol represent mean pointing response for that target. Error bars correspond to $\pm 1 S D$.

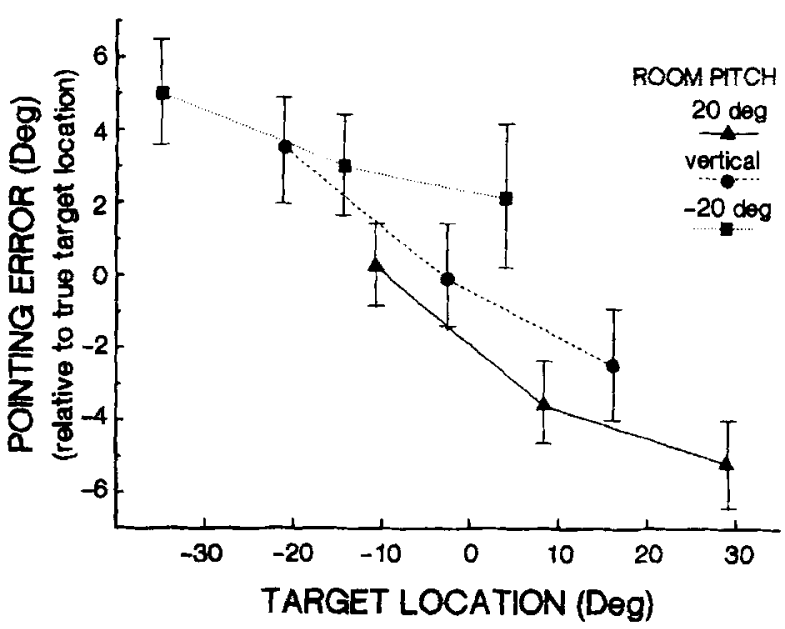

Figure 5. Mean pointing errors for targets in Experiment 2. For each room pitch, left, middle, and right data points correspond to the results for targets located below, at, and above the VPEL, respectively. Positive and negative errors indicate pointing above and below the intended target, respectively. Error bars correspond to $\pm 1 S E M$.

was elevated and depressed by upward and downward room pitch, respectively. A one-way (pitch direction) ANOVA revealed that the effect of pitch on VPEL was highly significant $[F(2,30)=176.52, p<.001]$. The mean pointing response for each of the nine targets is presented immediately adjacent to the symbol depicting target location. Pointing responses, on the average, were below the targets for upward room pitch, and above the targets for downward room pitch. In the vertical room condition, pointing was coincident with the VPEL target, above the lower target, and below the upper target.

Target-pointing accuracy was analyzed by first taking the difference between each pointing response and the intended target. These error scores were analyzed by a 3 (pitch direction) $\times 3$ (target) ANOVA. The main effects for pitch direction $[F(2,30)=25.41, p<.001]$ and target $[F(2,30)=25.63, p<.001]$ were both statistically significant. Figure 5 presents pointing errors for each pitch direction and target as a function of the elevation of the target relative to the subject's objective eye level. Subjects pointed below the targets with upward room pitch and above targets with downward room pitch. For a given room pitch, pointing errors also varied systematically with the distance of the targets from true eye level. Targets which were farthest above or below eye level produced the greatest under- and overpointing errors, respectively. The interaction of pitch direction and target level was also statistically significant $[F(4,60)=$ $2.97, p<.05]$. A planned post hoc analysis determined that in the room-vertical condition, pointing error for the upper target was significantly different from that for the lower target $[F(1,15)=38.57, p<.001]$. This result indicates that, even in the absence of a room pitch, the range of pointing responses was restricted relative to the range of actual target positions. 


\section{Discussion}

In Experiment 2, as in Experiment 1, VPEL was shifted in the direction of the room pitch- $8.4^{\circ}$ up for the upward room pitch and $14.3^{\circ}$ down for the downward room pitch. The range of VPEL values was therefore $22.7^{\circ}$, or $57 \%$ of the $40^{\circ}$ range of room pitches used. These percentage values for VPEL shifts approximate those found in Experiment 1 and in previous experiments (Ballinger, 1988; Matin \& Fox, 1989; Stoper \& Cohen, 1989).

On the average, subjects in Experiment 2 pointed below their VPEL in the case of upward room pitch and above their VPEL in the case of downward room pitch. Mean pointing level was elevated $4.8^{\circ}$ by the upward room pitch and depressed $11.3^{\circ}$ by the downward room pitch. The range of pointing responses was therefore $16.1^{\circ}$, or $71 \%$ of the $22.7^{\circ}$ change in VPEL values. These results are very similar to those of Experiment 1.

It is important to note that, even with the room vertical, subjects pointed somewhat low for high targets and somewhat high for low targets. This is apparent in the center panel of Figure 4, which shows that in the roomvertical case, subjects adjusted their pointing responses through approximately $85 \%$ of the range between the upper and lower targets. Therefore some, but not all, of the pointing errors may be attributed to a compression of the range of open-loop pointing. The compression of open-loop pointing responses is similar to that reported by Nemire and Bridgeman (1987) in a study on saccadic and reaching accuracy, and it contributes to the tendency to point above VPEL with downward pitch and below VPEL with upward pitch. It is apparent from Figure 5, however, that this compression of pointing is not entirely responsible for the effect of room pitch on pointing accuracy. If it were, the plotted data would not be segregated into different curves according to room pitch, but would follow one function determined solely by the elevation of the target.

\section{GENERAL DISCUSSION}

The finding that visual pitch leads to target-pointing errors is consistent with the hypothesis that the pitchroom changes the perception of object location. Subjects, when pointing at the incorrectly perceived target location, reveal the direction in which perceived location has been shifted. The change in VPEL cannot be attributed to a straight-ahead shift (Harris, 1974), because errors in open-loop pointing were found for targets whether or not their location was defined as being at eye level.

The ranges of pointing responses in Experiments 1 and 2 are larger than those obtained by Ballinger (1988; Cohen \& Ballinger, 1989), who reported pointing errors of approximately half the size of the observed VPEL shifts. A possible source of the apparent discrepancy between her experiment and ours is the fact that, unlike in the present studies, in which the pitch axes were at the center of the pitchrooms, Ballinger's smaller pitchbox pivoted around the center of its back wall. For this reason, rotation of the pitchrooms in the present studies was accompanied by vertical translations of the back wall, which did not occur in Ballinger's study. Stoper and Cohen (1989) have shown that such translations are likely to alter the VPEL independently of pitch. At present, however, it cannot be determined whether the occurrence of vertical translations in our studies and their absence in Ballinger's is the reason for the differences in reported pointing errors.

The change in perceived location induced by the pitchroom appears similar to that caused by looking through a weak prism. With prisms, subjects make initial pointing errors in the direction of the displacement, and when visual feedback about these errors is provided, they rapidly eliminate their errors. Following removal of the prism (or, in the present context, after the pitchroom is returned to the vertical orientation), subjects display negative aftereffects- pointing errors opposite those made initially. The target-reaching aftereffect in the first experiment was not, however, accompanied by a shift in postpitch VPEL measures. Thus, it appears that the adaptation following visual feedback in the pitchroom is not visual, but, rather, is attributable to changes in the target-reaching limb. This pattern of results is congruent with many examples of prism adaptation in which it is the felt position of the limb that undergoes modification (e.g., Harris, 1980).

Contrary to the prism situation, the magnitude of pointing errors caused by the pitchroom was much less than the VPEL shifts. Therefore, it is possible that, in addition to changing the perceived visual location of objects, room pitch shifts the perceived orientation of the whole body. If subjects misperceive both the location of an object and the orientation of the body, their pointing errors will be relatively small because the two effects will counteract one another. Nemire and Cohen (1993) demonstrated that the pitchroom influences perceived orientation of the body when subjects attempted to adjust body angle either to vertical or to a $45^{\circ}$ slant in a pitchroom set at various orientations. The size of the effect of pitch on body orientation responses was somewhat smaller, however, than that reported here and elsewhere for the effect of pitch on VPEL.

An alternative interpretation of the failure of pointing errors to correspond to VPEL shifts is that the perceptual and motor systems are not sharing the same map. Evidence for such a mismatch comes from Bridgeman, Lewis, Heit, and Nagle (1979), who found that, although subjects did not perceive the displacement of a target that occurred during a saccade, they pointed accurately at its new location. This result was interpreted to mean that the motor system that was used for the pointing response had access to information unavailable to the perceptual system involved in perceived motion. A similar explanation was offered for the finding that induced motion resulted in distortions of the perception of movement of a target, but did not affect pointing accuracy (Bridgeman, Kirch, \& Sperling, 1981). Thus, it is possi- 
ble that the effects of the pitchroom work in much the same way by distorting the perceived location of objects, while having only a weak effect on pointing accuracy.

\section{REFERENCES}

BALLINGER, C. J. (1988). The effects of a pitched field orientation on hand/eye coordination. Unpublished master's thesis, Naval Postgraduate School, Monterey, CA.

Bridgeman, B., Kirch, M., \& Sperling, A. (1981). Segregation of cognitive and motor aspects of visual function using induced motion. Perception \& Psychophysics, 29, 336-342.

Bridgeman, B., Lewis, S., Heir, G., \& Nagle, M. (1979). Relation between cognitive and motor-oriented systems of visual position perception. Journal of Experimental Psychology: Human Perception \& Performance, 5, 692-700.

Cohen, M. M., \& Ballinger, C. J. (1989). Hand-eye coordination is altered by viewing a target in a pitched visual frame [Abstract]. Aviation, Space, \& Environmental Medicine, 60, 477.

DichGans, J.. \& BRANDT, T. (1978). Visual-vestibular interactions: Effects on self-motion perception and postural control. In R. Held, H. Leibowitz, \& H.-L. Teuber (Eds.), Handbook of sensory physiology: Vol. 8. Perception (pp. 755-807). New York: Springer-Verlag.

DieTZEL, H (1924). Untersuchungen über die optische Lokalisation der Mediane [A study of the visual localization of the median]. Zeitschrift für Biologie, 80, 289-316.

EBENHolTZ, S. M. (1977). Determinants of the rod and frame effect: The role of retinal size. Perception \& Psychophysics, 22, 531-538.

EBEnholTz, S. M. (1985). Absence of relational determination in the rod-and-frame effect. Perception \& Psychophysics, 37, 303-306.

HARRIS, C. S. (1974). Beware the straight-ahead shift-a nonperceptual change in experiments on adaptation to displaced vision. Perception, $3,461-476$.

HARRIS, C. S. (1980). Insight or out of sight?: Two examples of perceptual plasticity in the human adult. In C. S. Harris (Ed.), Visual coding and adaptability (pp. 95-149). Hillsdale, NJ: Eribaum.

Harris, C. S., \& Gilchrist, A. (1976, April). Prism adaptation with- out prisms: A nonvisual change with implications about plasticity in the human visual system. Paper presented at the meeting of the Association for Research in Vision and Ophthalmology, Sarasota, FL. Howard, I. P. (1982). Human visual orientation. New York: Wiley. MATIN, L., \& Fox, C. R. (1989). Visually perceived eye level and perceived elevation of objects: Linearly additive influences from visual field pitch and from gravity. Vision Research, 29, 315-324.

MATIN, L., \& LI, W. (1992). Visually perceived eye level: Changes induced by a pitched-from-vertical 2-line visual field. Journal of Experimental Psychology: Human Perception \& Performance, 18, 257. 289.

Nemire, K, \& BRidgeman, B. (1987). Oculomotor and skeletal motor systems share one map of visual space. Vision Research, 27, 393400 .

Nemire, K., \& Cohen, M. M. (1993). Visual and somesthetic influences on postural orientation in the median plane. Perception \& $P_{s y}$ chophysics, 53, 106-116.

RoElofs, C. O. (1935). Optische Lokalisation [Visual localization]. Archiv für Augenheilkunde, 109, 395-415.

STOPER, A. E., \& COHEN, M. M. (1989). Effeet of structured visual environments on apparent eye level. Perception \& Psychophysics, 46, 469-475.

WELCH, R. B. (1978). Perceptual modification: Adapting to altered sensory environments. New York: Academic Press.

Witkin, H. A., \& Asch, S. E. (1948). Studies in space orientation: IV. Further experiments on perception of the upright with displaced visual fields. Journal of Experimental Psychology, 38, 762-782.

\section{NOTE}

1. The term reaching refers to bringing the hand or other body part into contact with, or in close proximity to, the target; pointing refers to aiming the hand, other body part, or (as in Experiment 2) a manual pointer in the direction of the distant target.

(Manuscript received November 29, 1994; revision accepted for publication August 3, 1995.) 